\title{
Soziale Netzwerke und Behinderung - Zugang und Stabilisierung der Einbindung in den allgemeinen Arbeitsmarkt
}

\section{Stefan Zapfel, Nancy Reims und Mathilde Niehaus}

\section{Überblick}

- Die arbeitsmarktbezogene Behinderungs- und Rehabilitationsforschung verzichtet bisher weitestgehend auf die Verwendung von Netzwerktheorien. Ihr Analyse- und Erklärungspotenzial wurde daher auf diesem Gebiet bei weitem noch nicht ausgeschöpft.

- Behinderungen stehen jedoch in engem Zusammenhang mit Genese und Stabilität von Netzwerken, die ihrerseits mit Zugang und Kontinuität von Beschäftigungsverhältnissen korrespondieren.

- Wohlfahrtsstaatliche Regelungen und Institutionen (z. B. Schwerbehindertenvertretung, Betriebliches Eingliederungsmanagement, Zentrale Auslands- und Fachvermittlung der Bundesagentur für Arbeit, Einrichtungen der beruflichen Rehabilitation) bieten Menschen mit Behinderung die Möglichkeit, Netzwerkkontakte (wieder) aufzubauen oder zu erweitern und Anschluss an den Arbeitsmarkt zu finden bzw. diesen aufrechtzuerhalten.

S. Zapfel $(\bowtie) \cdot$ N. Reims

Nürnberg, Deutschland

E-Mail: stefan.zapfel@ifes.uni-erlangen.de

N. Reims

E-Mail: nancy.reims@iab.de

M. Niehaus

Köln, Deutschland

E-Mail: mathilde.niehaus@uni-koeln.de 
- Inwiefern eine Eingliederung ins Erwerbssystem gelingt, hängt wesentlich von der Zugänglichkeit solcher Hilfen, dem Engagement sozialstaatlicher Akteure, deren Zusammenarbeit, der Teilnahmemotivation behinderter Menschen sowie dem individuellen Bildungshintergrund und sozialen Rückhalt ab.

\section{$1 \quad$ Einleitung}

Sozialwissenschaftliche Studien und amtliche Statistiken belegen immer wieder die vergleichsweise ungünstigen Beschäftigungsaussichten von Menschen mit Behinderung in Deutschland (Eichhorst et al. 2010, S. 7; Engels et al. 2017, S. 166 ff.; von Kardorff et al. 2013, S. 7 ff.; Pfaff 2012, S. 235 f.; Rauch 2005, S. 28 ff.). Menschen mit Behinderung profitieren deswegen auch in geringerem Umfang von den materiellen und latenten Funktionen, die Erwerbsarbeit leistet (Jahoda 1983). Zu den materiellen Funktionen gehören vor allem die Aneignung finanzieller Mittel und der Zugang zum Sozialversicherungssystem. Die latenten Funktionen umfassen u. a. vorgegebene zeitliche Strukturen, das Vorliegen einer gemeinschaftlichen Zielsetzung, die Zuweisung von sozialem und beruflichem Prestige, die Ausübung einer als sinnhaft erfahrenen Tätigkeit sowie die Herstellung und Aufrechterhaltung sozialer Kontakte. Zugangsdefizite zu den latenten Funktionen von Erwerbsarbeit zählen zu den wesentlichen Erklärungsfaktoren für die durchschnittlich schlechtere Gesundheitsverfassung von Arbeitslosen (Batinic et al. 2010; Jahoda 1982).

Angesichts der schwierigen Beschäftigungslage behinderter Menschen existiert in Deutschland eine Vielzahl arbeitsmarktpolitischer Maßnahmen, die das Ziel haben, die Erwerbssituation von Menschen mit Behinderung zu verbessern, $\mathrm{zu}$ erhalten oder zu stabilisieren. Neben allgemeinen Leistungen der Arbeitslosenversicherung im Bereich der Berufsberatung und Arbeitsvermittlung, die auch Menschen ohne Behinderung zustehen (Schröder et al. 2009, S. 13; Wuppinger und Rauch 2010, S. 8), gehören hierzu u. a. die Beschäftigungsquote von fünf Prozent ab einer Betriebsgröße von 20 Personen (§ 154 SGB IX, 2018), der erweiterte Kündigungsschutz ( $\$ 168-175$ SGB IX, 2018), Eingliederungszuschüsse, die Arbeitgebern gegebenenfalls für die Anstellung behinderter Menschen gewährt werden ( $\$ 88$ SGB III), Leistungen zur Teilhabe ( $\$ 50$ SGB IX, 2018), die Bereitstellung von Arbeitsassistenzen (§ 185 SGB IX), Unterstützte 
Beschäftigung ( 55 SGB IX, 2018), die Probebeschäftigung ( $\$ 46$ SGB III), das betriebliche Eingliederungsmanagement (BEM; § 167 SGB IX, 2018) oder auch Bildungsmaßnahmen der beruflichen Rehabilitation (Niehaus et al. 2012, S. 50 ff.; Weber und Weber 2015, S. 265 f.). Letztgenannte werden danach unterschieden, ob sie sich speziell an junge Berufsanfänger (Ersteingliederung) (Reims et al. 2016, S. 1) oder berufserfahrene Menschen mit Behinderung (Wiedereingliederung) richten (Reims et al. 2017).

Manche dieser Leistungen erfordern das Vorliegen einer Behinderung nach $\S 2$ SGB IX (2018), der auch diesem Beitrag als Definitionsgrundlage dient und nach der Menschen dann als behindert gelten, wenn sie ,[...] körperliche, seelische, geistige oder Sinnesbeeinträchtigungen haben, die sie in Wechselwirkung mit einstellungs- und umweltbedingten Barrieren an der gleichberechtigten Teilhabe an der Gesellschaft mit hoher Wahrscheinlichkeit länger als sechs Monate hindern können“ (§ 2 SGB IX, 2018). Andere Leistungen setzen eine amtlich anerkannte Schwerbehinderung (oder rechtliche Gleichstellung) voraus (SBG IX, Teil 3, $\S 151 \mathrm{ff}$., 2018), obwohl der amtlich verbürgte Behinderungsgrad nur bedingt für das Ausmaß der gesellschaftlichen Teilhabeeinschränkung aussagekräftig ist (Benitez-Silva et al. 2004; Rohrmann 2012, S. 475).

Die Bereitstellung wohlfahrtsstaatlicher Leistungen kann neben der Erzeugung von Inaktivitätsfallen (Famira-Mühlberger et al. 2015, S. 18) dazu beitragen, die unter Arbeitslosen in der Regel stärker in Mitleidenschaft gezogene Widerstandsfähigkeit gegen Erkrankungen zu erhöhen, geeignete Coping-Strategien zu entwickeln, wenn bereits eine Behinderung oder Erkrankung vorliegt (Potts 2005), und den Zugang zu Erwerbsarbeit zu erleichtern (Granovetter 1973). Soziale Netzwerke von Personen, Gruppen und Institutionen (Borgatti et al. 2018, S. 2) können ähnliche Wirkungen entfalten, aber trotz ihrer Bedeutung für Gesundheit, Bildung und Beschäftigung werden sie in der behinderungsbezogenen Gesundheits- und Rehabilitationsforschung bisher noch relativ selten berücksichtigt. Das gilt auch für die Methoden und Theorien der Netzwerkforschung (von Kardorff 2010).

Ziel des Beitrags ist es, diese Lücke in der Fachdiskussion einzugrenzen, indem er theoretisch und empirisch Ansatzpunkte für die Anwendung von Netzwerk- und Sozialkapitalansätzen auf dem Gebiet der Arbeitsmarkteingliederung behinderter Menschen aufzeigt und kritisch die bestehenden Forschungsdefizite in diesem Feld offenlegt. Im Fokus stehen dabei formale, informelle und institutionelle Beziehungsgeflechte, die aus ihnen resultierenden Unterstützungskapazitäten sowie mögliche Netzwerkänderungen durch einen Behinderungseintritt und die Auswirkungen, die solche Netzwerktransformationen auf die Beschäftigungssituation und die Erwerbschancen haben. 


\section{$2 \quad$ Behinderung und Arbeitsmarktintegration}

Für Deutschland wurden die vergleichsweise ungünstigen Bildungs- und Beschäftigungschancen von Menschen mit Behinderung bereits vielfach belegt (Eichhorst et al. 2010; Engels et al. 2017; Pfaff 2012; Rauch 2005; von Kardorff und Ohlbrecht 2013). Behinderte Menschen erreichen in der Bundesrepublik ein insgesamt niedrigeres Bildungsniveau als Personen, die keine Behinderung haben. Das gilt speziell für diejenigen, die schon frühzeitig im Förderschulbereich segregiert werden, der seinerseits häufig keinen Hauptschulabschluss bereithält (Klemm 2015). Jugendliche in Förderschulen verlassen deshalb ihre Bildungseinrichtung oft ohne Hauptschulabschluss, wodurch sich die Chancen auf einen Ausbildungsplatz entsprechend reduzieren (Niehaus et al. 2012). Sofern sie dennoch Zugang zum Ausbildungssystem erhalten, werden sie in der Regel weiterhin in Sonderbereichen mit geringer betrieblicher Anbindung (weiter-) qualifiziert (Reims et al. 2016). Im Verbund mit einer Reihe weiterer, vor allem stigmatisierungsbedingter Faktoren (Einstellungsvorbehalte auf Arbeitgeberseite, Verdacht auf Leistungsminderung, Angst vor hohen Fehlzeiten, erschwerte Kündigung, aber auch Mobilitätseinschränkungen u.ä.m.) (z. B. Niehaus und Bauer 2013, S. 12 f.; von Kardorff et al. 2013, S. 37; Rauch 2005, S. 32; Wansing und Westphal 2014, S. 41), schmälert diese bildungsinstitutionelle Streckenführung die Beschäftigungsaussichten, die Menschen mit Behinderung in Deutschland haben. Diese dokumentieren sich in niedrigeren Erwerbs- und höheren Arbeitslosenquoten als bei Menschen ohne Behinderung, selbst in Phasen wirtschaftlichen Aufschwungs (Bundesagentur für Arbeit 2018; Niehaus und Bauer 2013, S. 32), längeren Arbeitslosigkeitsepisoden, geringeren Löhnen und der häufigeren Besetzung von Berufspositionen, die sich unter dem erreichten Qualifikationsniveau befinden (Weller 2017).

Diverse arbeitsmarktpolitische Instrumente haben die Aufgabe, Benachteiligungen behinderter Menschen auf dem Arbeitsmarkt zu bekämpfen und zu kompensieren. Hierzu gehören alle schon genannten Maßnahmen, die in Bezug auf das Verhältnis zwischen Arbeitgebern und Menschen mit Behinderung entweder eine beziehungsgenerierende oder eine beziehungsstabilisierende Funktion übernehmen (sollen) und damit Netzwerkrelevanz haben. Unter den speziell auf Menschen mit Behinderung zugeschnittenen Instrumenten wirken die Beschäftigungsquote ab 20 Mitarbeitern, die Probebeschäftigung sowie Leistungen der beruflichen Rehabilitation im Wesentlichen beziehungsgenerierend, während der erweiterte Kündigungsschutz und das Betriebliche Eingliederungsmanagement in erster Linie beziehungsfestigend sind. Arbeitsplatzanpassungen, 
die Bereitstellung von Arbeitsassistenzen und Unterstützte Beschäftigung fördern beide Bereiche, indem sie sowohl die Beschäftigungsaufnahme als auch die Verstetigung von Arbeitsverhältnissen erleichtern.

\section{Soziale Netzwerke von Menschen mit Behinderung}

Verschiedene Studien, darunter auch der Teilhabebericht der Bundesregierung über Lebenslagen von Menschen mit Beeinträchtigungen, zeigen, dass behinderte Menschen häufig weniger soziale Kontakte aufweisen als andere Gruppen (Engels et al. 2017; Forrester-Jones et al. 2006; Morgan et al. 1984; Pfaff 2012, S. 234; Schröttle et al. 2014, S. 24). Sie haben kleinere Netzwerke und gehen seltener neue lebensweltliche Beziehungen ein (Forrester-Jones et al. 2006; Schröttle et al. 2014). Darüber hinaus kann der Eintritt einer Behinderung von einem Verlust an Beziehungen und Netzwerken begleitet werden (von Kardorff 2010). Er kann einerseits dazu führen, dass die bisherige Tätigkeit im Erwerbssystem nicht mehr ausgeübt werden kann und sich parallel dazu die bisherigen Kontakte in die Arbeitswelt lockern oder auflösen (Lang 2003, S. 181). Andererseits kann ein Behinderungseintritt auf lebensweltliche Beziehungen erodierend wirken, zumal er unter Umständen neue Belastungen auch für den sozialen Nahkreis mit sich bringt und bestehende Hilfebedarfe partiell die in der Privatsphäre verfügbaren Unterstützungskapazitäten übersteigen (von Kardorff 2010). Gefährdet sind vor allem bereits losere, emotional weniger stark unterfütterte Bindungen. Gleichzeitig kann der Aufbau neuer Kontakte aus Barrieregründen oder stigmatisierungsbedingt erschwert sein (Pfaff 2012; Schröttle et al. 2013, S. 24), sodass das soziale Isolationsrisiko steigt (Morgan et al. 1984, S. 495), mit dem aber wiederum die Zugangschancen zu professionellen Unterstützungsangeboten sinken (von Kardorff 2010). Frauen mit Schwerbehinderung sind hiervon besonders bedroht ${ }^{1}$ (Niehaus 1993; Niehaus und Bauer 2013).

Allerdings kommt es wegen einer Behinderung bei weitem nicht immer $\mathrm{zu}$ einer Auflösung von lebensweltlichen Beziehungen. Häufig bleibt es bei einer Änderung in den Beziehungsverhältnissen, die jedoch gravierend sein

\footnotetext{
${ }^{1}$ In der behinderungsbezogenen Inklusions- und Teilhabeforschung werden kumulative, an unterschiedliche soziale Merkmale gebundene Benachteiligungen intensiv unter dem Begriff Intersektionalität behandelt. Sie meint eine Exklusionswirkung, die aus der negativen Bewertung mehrerer personenbezogener Merkmale wie Geschlecht, Behinderung oder ethnischer Zugehörigkeit hervorgeht (Weinbach 2014).
} 
können. Solche Transformationen reichen von Verschiebungen in der familialen Arbeitsteilung und der Umstellung von Routinen und Zeitplänen in der Alltagsbewältigung über neue soziale Anforderungen an Mobilität und Affektivität bis hin zu erweiterten finanziellen Bedarfen (von Kardorff 2010). Netzwerkschrumpfungen gehen manchmal mit einer höheren Dichte des verbleibenden Netzwerks einher, wie empirische Arbeiten über kognitiv beeinträchtigte und schwerbehinderte Menschen zeigen (Forrester-Jones et al. 2006).

Mit den bisherigen Ausführungen ist bereits angedeutet, dass Netzwerke behinderter Menschen in Art und Qualität erheblich divergieren und mitunter spezifischen Charakter haben (Engels et al. 2017). Unterschiede bestehen etwa im Hinblick auf den Institutionalisierungsgrad (informell oder formal), ihre gesellschaftliche Verortung (z. B. Lebens- oder Arbeitswelt), den Entstehungskontext (etwa vor oder nach Behinderungseintritt oder Entstehungszweck), die Funktion (emotional, instrumental, informationsbezogen) oder die jeweiligen Unterstützungskapazitäten (Chronister et al. 2008).

Informelle Netzwerke bieten in der Regel informelle Hilfen. Sie ergeben sich einerseits aus der lebensweltlichen Einbettung in Familien, Freundschafts- und Bekanntenkreisen und entstehen großteils außerhalb von Erwerbszusammenhängen. Andererseits gehen informelle Beziehungen auch aus dem Erwerbssystem hervor und erzeugen soziale Bindungen zu Vorgesetzten, Untergebenen und Kollegen in unterschiedlicher Intensität (Knox und Parmenter 1993). Die Beziehungen können sowohl aus vertikalen Strukturen, die sich als hierarchische Struktur und somit zwischen den Mitarbeitern und ihren direkten Vorgesetzten verläuft, beschreiben lassen, als auch aus der horizontalen Struktur, welche die sozialen Beziehungen der Mitarbeiter untereinander beschreibt, entstehen (Badura 2008). Aus beiden Bereichen ergeben sich Ressourcen sozialer Unterstützung, die auch die Eingliederung in den Arbeitsmarkt und Möglichkeiten gesellschaftlicher Teilhabe begünstigen können (Granovetter 1995, S. 48). Allerdings kann ein mit Behinderung in Zusammenhang stehender erhöhter Unterstützungsbedarf zugleich neue (informelle) Abhängigkeiten erzeugen, die dem Selbstbestimmungsversprechen für Menschen mit Behinderung, wie es etwa in der UN-Behindertenrechtskonvention (UN-BRK), im Behindertengleichstellungsgesetz oder im Bundesteilhabegesetz zum Ausdruck kommt (vgl. z. B. Kastl 2017, S. 229; Lewicki 2014, S. 12; Welti 2005, S. 23 ff.), zuwiderlaufen (Bundesministerium für Arbeit und Soziales [BMAS] 2011, S. 53; Schröttle et al. 2013, S. 75).

Neben informellen Beziehungen sind auch formale, zum Teil rechtlich vorgeschriebene und regulierte Beziehungen zum Arbeitgeber bzw. im Betrieb (etwa durch das Betriebliche Eingliederungsmanagement oder die Schwerbehindertenvertretung (SBV)) wesentlich für den Zugang und die Aufrechterhaltung von 
Beschäftigungsverhältnissen. Gleiches gilt für Verbände und andere Interessensvertretungen von Menschen mit Behinderung oder Einrichtungen der beruflichen Rehabilitation (Bundesagentur für Arbeit, Rentenversicherung, Berufsförderungswerke usw.), die Beschäftigungsmöglichkeiten für behinderte Menschen schaffen, stärken oder sichern sollen. Anders als in lebensweltlichen Zusammenhängen ist der Eintritt einer Behinderung dort kein Ereignis, das das Risiko von Beziehungsverlusten erhöht, sondern im Gegenteil gerade ausschlaggebend für den Aufbau von Kontakten zu solchen Einrichtungen und Gruppen, die Hilfe bieten (vgl. Borgatti et al. 2018, S. 4).

Eine der normativen Grundanforderungen, die in Deutschland sowohl in Bezug auf informelle als auch im Hinblick auf formale Unterstützung zur Aufnahme und Aufrechterhaltung von Beschäftigungsverhältnissen behinderter Menschen gestellt werden, besteht hierbei darin, den Wünschen und Ansprüchen der Unterstützungsempfänger Rechnung zu tragen und autoritäre Abhängigkeiten zu vermeiden (Chronister et al. 2008).

\section{Die Rolle sozialer Netzwerke bei der Arbeitsmarktintegration von Menschen mit Behinderung}

Soziale Beziehungen und Netzwerke sind sowohl informell als auch institutionell für die Eingliederung in den Arbeitsmarkt von Bedeutung (Brucker 2015). Das gilt auch für Menschen mit Behinderung. Zugang und Nutzung sind dabei sehr unterschiedlich. Inwiefern die beiden Varianten auf die Einbindung behinderter Menschen ins Erwerbssystem Einfluss nehmen und welche Rolle sie sozial- und arbeitsmarktpolitisch spielen, wird im Folgenden erläutert.

\subsection{Informelle Beziehungen zur Förderung der Teilhabe am Arbeitsleben}

Die Arbeitsmarktforschung hat wiederholt auf die Bedeutung lebensweltlicher und beruflicher Beziehungen und Netzwerke für den Zugang zu Erwerbsarbeit und Karriere hingewiesen (Granovetter 1973, S. 1371 ff.; Granovetter 1995, S. 4 ff.). Dabei wird meist auf die „Unvollkommenheit“ von Arbeitsmärkten Bezug genommen (z. B. Lin 2009, S. 20): Entgegen den verbreiteten Annahmen der neoklassischen Arbeitsmarkttheorie besäßen weder Arbeitgeber noch (potenzielle) Arbeitnehmer alle relevanten Informationen, die bei der Stellen- bzw. 
Bewerbersuche benötigt werden (Hinz und Abraham 2008, S. 51 ff.). Netzwerke übernähmen in diesen Zusammenhang die Funktion, bestehende Informationslücken informell zu schließen. Da sich aber Zugang und Qualität der Netzwerke voneinander unterschieden, seien die Möglichkeiten hierfür ungleich verteilt. Die Beschäftigungschancen und Karriereaussichten gestalteten sich umso besser, je größer und heterogener die Netzwerke etwa im Hinblick auf Berufszugehörigkeiten oder den sozialen Status seien (Diewald und Sattler 2010; Granovetter 1995, S. 12 ff.). Mit Größe und divergenter Zusammensetzung steige die verfügbare Informationsvielfalt, jedoch nehme gleichzeitig die Zahl schwacher sozialer Bindungen im Netzwerk zu; schwache Bindungen kämen dem arbeitsmarktbezogenen Informationsgehalt zugute, enge Bindungen begünstigten hingegen Unterstützungsmöglichkeiten bei der Alltagsbewältigung und Coping (Potts 2005).

Nun stehen Gesundheit, Erwerbsarbeit, Behinderung, gesellschaftliche Teilhabe sowie die Verfügbarkeit und Einbindung in Netzwerke miteinander in engem Zusammenhang (Niehaus 1993). Da Menschen mit Behinderung in der Regel kleinere Netzwerke mit größerer Dichte aufweisen und seltener als in anderen Gruppen neue Kontakte dazukommen, bieten ihnen ihre vorhandenen Beziehungen weniger Gelegenheiten zur (Re-)Integration ins Erwerbssystem. Hinzu kommt im Falle einer im Lebenslauf auftretenden Behinderung bei gleichzeitigem Arbeitsplatzverlust, dass bestehende Kontakte ins Arbeitsleben verloren gehen und damit wichtige Informations- und Unterstützungskanäle entfallen, die den Wiedereintritt in den Arbeitsmarkt erleichtern könnten. Informelle Beziehungen helfen demnach Menschen mit Behinderung insgesamt in geringerem Umfang beim Arbeitsmarktzugang und Karrierewegen als es bei nicht-behinderten Menschen der Fall ist. Anders gestaltet sich die Situation bei institutionellen Beziehungen und Netzwerken.

\subsection{Institutionelle Beziehungen zur Förderung der Teilhabe am Arbeitsleben}

Institutionelle Netzwerke und Beziehungen von und für Menschen mit Behinderung sind in Deutschland vielfältig und wurden zum Teil bewusst eingerichtet, um behinderte Menschen bei der Eingliederung in den Arbeitsmarkt zu unterstützen. Andere wurden zu nicht-arbeitsbezogenen Zwecken geschaffen, helfen dem Personenkreis aber nichtsdestoweniger, Zugang ins Erwerbsleben zu finden oder bestehende Beschäftigungsverhältnisse zu stabilisieren. 
Von besonderer Bedeutung sind in diesem Zusammenhang Interessenverbände und Vereine behinderter Menschen, wohlfahrtsstaatliche Bestimmungen, die die Einbindung von schwerbehinderten Menschen im Betrieb betreffen (etwa Schwerbehindertenvertretung oder Betriebliches Eingliederungsmanagement), und sozialstaatliche Einrichtungen der beruflichen Rehabilitation und Arbeitsvermittlung, die speziell Menschen mit Behinderung bei der Arbeitsmarkteinbindung helfen. Letztgenannte Institutionen nutzen gezielt professionelle Netzwerke, um entsprechende Programme und Maßnahmen der Arbeitsmarktintegration umzusetzen.

\subsubsection{Interessenverbände und Vereine}

Behindertenorganisationen sind zentrale Akteure der Interessensvertretung von Menschen mit Behinderung und bieten ihren Mitgliedern in unterschiedlicher Weise Unterstützung in Alltag und Lebensführung. Sie sind nicht speziell auf die Eingliederung behinderter Menschen in den Arbeitsmarkt gerichtet, fördern aber durch Beratungsangebote und die Verbreitung von Stellenanzeigen beispielsweise per Newsletter die Beschäftigungsaufnahme ihrer Mitglieder.

Neben den etablierten Verbänden gibt es diverse Selbsthilfezusammenschlüsse für bestimmte Behinderungsarten und Erkrankungen, die sich in ihrem Organisationsgrad erheblich voneinander unterscheiden. Die BAG Selbsthilfe ist die Dachorganisation von 113 Organisationen behinderter und chronisch kranker Menschen und ihren Angehörigen. Sie vertritt als bundesweiter Zusammenschluss unabhängig von parteipolitischen oder konfessionellen Bindungen die Interessen der Gesamtheit der behinderten und chronisch kranken Menschen als freier Verband. Unter ihrem Dach haben sich auch die in den 1990er Jahren gegründeten Netzwerke von und für Frauen mit Behinderung organisiert, die den spezifischen Belangen von Mädchen und Frauen mit Behinderung mehr Gehör verschaffen wollen (Niehaus 2001). Ihre Aktivitäten und Forderungen sind u. a. in die Gesetzgebung zum Neunten Sozialgesetzbuch eingeflossen, unterstützen ihre Mitglieder also nicht nur individuell, sondern auch politisch durch Einflussnahme auf die Bundes- und Landesgesetzgebung.

Um die politischen Interessen behinderter Menschen durchzusetzen, streben Behindertenorganisationen in Deutschland Konsens- und Kompromissbildungen an. Dabei gehen sie Bündnisse mit Gewerkschaften und Verbänden der Freien Wohlfahrtspflege ein, mit denen sie ihre Durchsetzungschancen zu verbessern suchen (Hammerschmidt 1992). Die Betätigungsfelder sind unterschiedlich und betreffen neben Bildung auch die politische Teilhabe, Fragen der sozialen Sicherung, Arbeit und Beschäftigung u.a.m. (Engels et al. 2017). 
Allerdings wirken Behindertenorganisationen in zweierlei Weise selektiv (Bengtsson und Datta Gupta 2017): Zum einen sind sie häufig auf bestimmte Gruppen von Menschen mit Behinderung und chronischen Erkrankungen ausgerichtet, zum anderen sind es vorzugsweise höher gebildete Menschen mit Behinderung, die in diesen Verbänden organisiert sind und Zugang zu Informationen und Hilfen erhalten. So wichtig die Behindertenorganisationen also für die Gestaltung der Sozialpolitik und den Beschäftigungszugang in der Bundesrepublik auch sind, ihr Wirkungsradius sollte in einer objektiven Analyse nicht überschätzt werden.

\subsubsection{Schwerbehindertenvertretung (SBV)}

Die SBV ist eine gewählte Interessenvertretung der schwerbehinderten und ihnen gleichgestellten Menschen in Betrieben der Privatwirtschaft und Verwaltungen des öffentlichen Dienstes und in Deutschland fester Bestandteil des betrieblich-formalen Struktur- und Beziehungsgefüges. Sie hat Einfluss auf die betriebliche Beschäftigungskontinuität behinderter Menschen und deren soziale Einbettung in diesen Organisationen. Die Grundlagen der SBV-Arbeit sind in $\S \S$ 178 ff. SGB IX verankert und legen konkrete Rechte und Pflichten im Betriebsgeschehen fest. Die SBV ist auch für die Unterstützung von Beschäftigten zuständig, die nicht schwerbehindert, jedoch von Behinderung bedroht sind, etwa chronisch kranke Personen.

Die SBV unterstützt mit ihrer Expertise auf dem Gebiet betrieblicher Teilhabe bei Behinderung und chronischer Erkrankung den Betriebsrat und den Arbeitgeber bei Fragen der (Wieder-)Eingliederung. Sie hat durch ihre umfangreichen sozialrechtlichen Kenntnisse, die Bereitstellung vertrauensvoller Ansprechpartner, die Übernahme koordinierender Vermittlungsaufgaben und die proaktive Anbahnung betrieblicher Gesundheitsmaßnahmen eine Lotsenfunktion bei Fragen der betrieblichen Inklusion und Prävention (Kohl und Niehaus 2014). Informell trägt sie dazu bei, mögliche Arbeitgebervorbehalte bei der Einstellung behinderter Menschen (Verdacht auf verminderte Leistungsfähigkeit, auf Mobilitäts- und Flexibilitätseinschränkungen u. Ä.) abzubauen.

Die SBV fördert durch persönliche Beratung und Belegschaftsnähe auch das Vertrauensverhältnis zwischen den verschiedenen Akteuren im Betrieb (behinderte und chronisch kranke Menschen, Betriebsrat, Arbeitgeber, Betriebsarzt) (Deutsche Gesetzliche Unfallversicherung e. V. [DGUV] 2014). Vernetzung, Kooperation und Vertrauen zwischen den genannten Akteuren und zu den zuständigen Sozialversicherungsträgern sind zentrale Voraussetzungen für 
die erfolgreiche Durchführung des BEM und eine gelungene betriebliche Teilhabe (Niehaus und Vater 2014). Die Schwerbehindertenvertretungen sind somit wichtige Netzwerkpartner an der Schnittstelle zwischen behinderten Mitarbeitern, betrieblichen Organen (z. B. Betriebsrat), Arbeitgeberbeauftragten und externen Akteuren, zu denen auch Sozialversicherungsträger wie die Rentenversicherung oder die Krankenkassen gehören.

Durch die Unterzeichnung der Behindertenrechtskonvention der Vereinten Nationen (UN-BRK 2008) hat sich Deutschland mit der Ratifizierung 2008 dazu verpflichtet, die volle und wirksame Teilhabe von Menschen mit Behinderungen in allen gesellschaftlichen Bereichen herzustellen. Die entsprechend der UN-BRK definierte Zielvision eines inklusiven Arbeitsmarktes erweitert auch die Aufgaben der Vertrauenspersonen der schwerbehinderten Beschäftigten. Diese gehen zunehmend über Kenntnisse arbeitsrechtlicher Vorgaben hinaus und erstrecken sich immer mehr auch auf querschnittliche Beratungsfunktionen, die Vernetzung und Wissensmanagement in sehr unterschiedlichen Bereichen erfordern (Kohl et al. 2015). Dadurch stehen die Schwerbehindertenvertretungen in der betrieblichen Prävention und Inklusion vor neuen Herausforderungen, die sich u. a. aus großräumigen gesellschaftlichen Veränderungen wie Digitalisierung und demografischem Wandel ergeben. Das Tätigkeitsspektrum der SBV sowie innerbetriebliche und externe Allianzen, die zum Erhalt der Beschäftigungsfähigkeit und Teilhabe beitragen können, sind Mittelpunkt der aktuellen Diskussion. Netzwerkanalytische Untersuchungen gibt es hierzu bisher nicht. Diese könnten jedoch klären, ob und wie die SBV Menschen mit Behinderung unter diesen neuen Voraussetzungen bei der Eingliederung unterstützt und welche Rolle Vernetzungen der SBV in diesem Zusammenhang spielen.

\subsubsection{Betriebliches Eingliederungsmanagement (BEM)}

Mit dem BEM nach $\S 167$ Abs. 2 SGB IX (2018) hat der Gesetzgeber eine Vorschrift geschaffen, die Arbeitgeber seit 2004 dazu verpflichtet, bei Beschäftigten, die innerhalb eines Jahres insgesamt länger als sechs Wochen erkrankt sind, zu klären, inwiefern die Arbeitsunfähigkeit überwunden, erneuter Arbeitsunfähigkeit vorgebeugt und der Arbeitsplatz erhalten werden kann. Gemeinsam mit der zuständigen Interessenvertretung - bei Vorliegen einer Schwerbehinderung mit der SBV - und soweit erforderlich mithilfe des Werk- oder Betriebsarztes, wird geprüft, welche Maßnahmen hierfür zu ergreifen sind. Weitere in das BEM einbezogene Akteure können die Rehabilitationsträger und gegebenenfalls das Integrationsamt sein. Die Teilnahme der Arbeitnehmer am BEM ist freiwillig. 
Kleinere und mittelständische Unternehmen setzen aus Mangel an Ressourcen und an betrieblichen Stellenalternativen seltener das BEM um als gröBere Betriebe (Niehaus et al. 2008). $\mathrm{Zu}$ regionalen Anwendungsdifferenzen, verwendeten BEM-Standards und zur Rolle von Netzwerken beim Zugang zum BEM liegen bisher keine repräsentativen Forschungsbefunde vor.

\subsubsection{Zentrale Auslands- und Fachvermittlung und Peer- Beratung}

Die Zentrale Auslands- und Fachvermittlung der Bundesagentur für Arbeit (ZAV) unterstützt sowohl Akademiker mit Schwerbehinderung bei der Suche nach einer qualifikationsadäquaten Beschäftigung als auch Arbeitgeber bei der Stellenbesetzung mit schwerbehinderten Hochschulabsolventen (Deutsches Studentenwerk 2013, S. 201). Sie übernimmt als hierfür eingerichtete Organisation eine wichtige Vermittlungsfunktion schwerbehinderter Menschen mit Hochschulabschluss in Beschäftigung und versucht zu diesem Zweck gezielt Kontakte mit diesem Personenkreis und zwischen schwerbehinderten Menschen und Arbeitgebern herzustellen.

Parallel dazu werden Selbsthilfeaktivitäten bei Fragen der beruflichen Teilhabe von Menschen mit Behinderung zunehmend unter der Perspektive des Stellenwertes von Peer-Beratungen (Beratung von geschulten Betroffenen für Betroffene) diskutiert. Hintergrund dafür ist, dass Peer-Beratung als Bestandteil professioneller Beratungsangebote durch Anhebung der Reha-Motivation und den stärkeren Selbstbestimmungsbezug in der Entscheidungsfindung nachweislich zu einer verbesserten beruflichen Teilhabe beitragen kann. In Deutschland werden Forderungen nach mehr Peer-Beratung zur beruflichen Teilhabe nicht zuletzt durch Aktionspläne zur Umsetzung der UN-BRK der Bundesländer und der Gesetzlichen Unfallversicherung gestützt, in denen Peer-Counseling bereits als Maßnahme aufgenommen ist (Niehaus und Saupe-Heide 2012).

Im Bundesteilhabegesetz kommt seit 2018 der Peer-to-Peer-Beratung als ergänzende unabhängige Teilhabeberatung besondere Bedeutung zu. Die gesetzliche Verankerung fußt u. a. auf den Erkenntnissen der wissenschaftlichen Fachgruppe RehaFutur, die hervorhebt, dass berufliche Teilhabechancen verbessert werden, wenn die Selbstbestimmung und Selbstverantwortung von Menschen mit Behinderung aktiv genutzt und durch entsprechende Strukturen gestärkt werden (Riedel et al. 2009). Ob die Peer-to-Peer-Beratung als qualitatives Merkmal von Netzwerkstrukturen von Menschen mit Behinderung bessere Handlungsmöglichkeiten bereithält als eine Beratung durch nicht-behinderte Personen oder das Fehlen einer solchen Beratung, bleibt für die Forschung noch zu klären. 


\subsubsection{Rehabilitationsspezifische Institutionen}

Eine weitere Art sozialer Netze behinderter Menschen ist rehabilitationsspezifisch und entspringt den institutionellen und professionalisierten Unterstützungsformen, die dazu dienen, die Wiedereingliederung behinderter Menschen in den Arbeitsmarkt zu erleichtern oder zu verfestigen. In diesem Zusammenhang werden (ähnlich wie im Fall der ZAV) nach sozialrechtlichen und verwaltungsspezifischen Vorgaben bewusst formale Beziehungen zwischen Menschen mit Behinderung auf der einen Seite und Rehabilitationsberatung, Arbeitsvermittlung, Fallmanagement und potenziellen Arbeitgebern auf der anderen geknüpft. Die Rehabilitationsträger (Bundesagentur für Arbeit, Rentenversicherung, Gesetzliche Unfallversicherung etc.), Integrationsfachdienste und Leistungserbringer stellen hierbei durch (Fremd-)Investitionen in Sozialkapital (Bourdieu 2005, S. 65) Netzwerke her, die Menschen mit Behinderung dabei helfen sollen, wieder in Beschäftigung zu kommen oder in Erwerbsarbeit zu bleiben. Absicht der hieran professionell beteiligten Akteure ist es, eine Brückenfunktion (,,bridging“) (Putnam 2000, S. 411) zu übernehmen, mit deren Hilfe behinderten Menschen eingliederungsrelevante Netzwerkkontakte zur Verfügung gestellt werden, die bis ins Beschäftigungssystem hineinreichen (von Kardorff 2010).

Der Zugang zu beruflicher Rehabilitation und den dort vorhandenen Netzwerkstrukturen ist allerdings beschränkt und steht nur denjenigen offen, die einen förmlichen Antrag auf Leistungen zur Teilhabe am Arbeitsleben (LTA) gestellt haben und auch als Rehabilitand anerkannt wurden (Reims et al. 2017). Die Maßnahmeplanung erfolgt in enger Abstimmung zwischen Leistungserbringern, Kostenträgern und Rehabilitanden. Die Leistungserbringer von Maßnahmen der beruflichen Rehabilitation sind flexibel in der Maßnahmegestaltung und zeichnen sich durch eine breite Palette an Unterstützungs- und Netzwerkstrukturen aus. Sie bieten unterschiedliche Berufsbildungsmöglichkeiten an, sind teilweise auf bestimmte Behinderungsarten spezialisiert, stellen reha-begleitend in unterschiedlichen Ausprägungen sozialpädagogische oder medizinische bzw. therapeutische Zusatzangebote bereit, ermöglichen oft eine stationäre Unterbringung und sind in vielfältiger Weise mit Betrieben und potenziellen Arbeitgebern vernetzt. Aus den Unterschieden in der Netzwerkstruktur der Leistungserbringer können sich auch Divergenzen im Reha-Erfolg ergeben. So erscheinen kleinere, zentral organisierte und in Bezug auf das Arsenal an Leistungserbringern diversifizierte Netze integrationsförderlicher als größere, dezentral organisierte und homogene Netzwerke, während die Betreuungskontinuität eher in letztgenannter Konstellation gewährleistet werden kann (Lorant et al. 2017). 
Nach Beendigung der Qualifizierungsphase folgt häufig eine Übergangsbetreuung. Sie dient dazu, den Bewerbungsprozess zu begleiten und die Integration in den neuen Beruf zu erleichtern. Liegt eine Schwerbehinderung (oder eine Gleichstellung) vor, kann auch der Integrationsfachdienst hinzugezogen werden, der sowohl den Betrieben als auch Menschen mit Behinderung beratend und unterstützend zur Seite steht (von Kardorff 2010).

Es gibt also in Deutschland bereits ein breites institutionelles und professionelles Netzwerk, an dem unterschiedliche Akteure beteiligt sind. Dennoch werden nach wie vor erweiterte Vernetzungsbedarfe in der Leistungserbringerlandschaft angemahnt, mit deren Behebung fortbestehende Schnittstellenprobleme beseitigt, die Zugänglichkeit zu den Leistungen verbessert, Koordinationslücken geschlossen, Versorgungskosten reduziert und Wiedereingliederungserfolge erhöht werden sollen (von Kardorff 2010).

Im Prozess der beruflichen Rehabilitation sind nicht nur institutionelle Netzwerke von Bedeutung. Häufig spielen auch informelle, lebensweltliche Beziehungen der Rehabilitanden eine wichtige Rolle, die in der Maßnahmeplanung berücksichtigt werden (Chronister et al. 2008). Hintergrund hierfür ist einerseits die prominent in der International Classification of Functioning, Disability and Health (ICF) entwickelte Vorgabe, den personenbezogenen Gesamtzusammenhang bei der Planung der beruflichen Re-Integration zu berücksichtigen (Escorpizo et al. 2011), andererseits der in zahlreichen Studien belegte Umstand, dass der Rückhalt und der Kontakt mit Familie und Freunden während der Teilnahme an Teilhabeleistungen am Arbeitsleben das Rehabilitationsergebnis maßgeblich beeinflussen (Chronister et al. 2008; McKenna und Power 2000; Potts 2005). Der Effekt kann sowohl positiv als auch negativ sein, je nachdem, ob soziale Unterstützung und Rückhalt gegeben sind oder fehlen (Zapfel 2015, S. 242).

\section{$5 \quad$ Fazit, Desiderate}

Mit dem Eintritt einer Behinderung steigt das Risiko, informelle und Teile der formalen Netzwerke zu verlieren, gleichzeitig aber auch die Chance, andere - vor allem professionelle - Netzwerkpartner zu gewinnen. Wohlfahrtsstaatliche Regelungen und Institutionen wie die SBV, das BEM, die ZAV oder Einrichtungen der beruflichen Rehabilitation bieten Menschen mit Behinderung die Möglichkeit, Netzwerkkontakte neu aufzubauen oder zu erweitern und wieder Anschluss an den Arbeitsmarkt zu finden bzw. diesen aufrechtzuerhalten. Inwiefern das und eine erfolgreiche Eingliederung ins Erwerbssystem gelingt, 
hängt von verschiedenen Faktoren - vor allem der formalen Zugänglichkeit, dem Engagement sozialstaatlicher Akteure und ihrer Zusammenarbeit, der Inklusionsbereitschaft der Arbeitgeber, der Teilnahmemotivation behinderter Menschen, deren Bildungshintergrund sowie dem lebensweltlichen Rückhalt - ab.

In der arbeitsmarktbezogenen Behinderungs- und Rehabilitationsforschung wird bisher weitestgehend auf die Anwendung von Netzwerkansätzen verzichtet. Das gilt sowohl in Bezug auf informelle Zugangswege zum Arbeitsmarkt als auch im Hinblick auf die Rolle von Interessenverbänden behinderter Menschen, innerbetriebliche Organisationseinheiten und Abläufe (wie SBV und BEM), die Bedeutung der ZAV, Angebote der Peer-to-Peer-Beratung und Einrichtungen der beruflichen Rehabilitation. Aufgabe dieses Beitrags ist es, mögliche Ansatzpunkte hierfür aufzuzeigen, dabei relevantes empirisches Material miteinzubeziehen, entsprechende Forschungslücken zu verdeutlichen und Anregungen für künftige Forschungsaktivitäten auf diesem Gebiet zu geben.

\section{Leseempfehlungen}

Chronister, J., Chou, C. C., Frain, M. \& da Silva Cardoso, E. (2008). The relationship between social support and rehabilitation related outcomes: A meta-analysis. Journal of Rehabilitation, 74 (2), S. 16-32. https://www. questia.com/library/journal/1G1-182034960/the-relationship-between-social-support-and-rehabilitation. Zugegriffen: 04. April 2018. Der Artikel behandelt die Rolle sozialer Unterstützung zunächst generell in Bezug auf Erkrankungsrisiken und im weiteren Verlauf hinsichtlich der Erfolgsaussichten rehabilitativer Maßnahmen. Er gibt einen ausführlichen Überblick über psychologische, sozial- und gesundheitswissenschaftliche Veröffentlichungen im englischsprachigen Raum zu diesem Thema.

von Kardorff, E., Ohlbrecht, H. \& Schmidt, S. (2013). Zugang zum allgemeinen Arbeitsmarkt für Menschen mit Behinderungen. Expertise im Auftrag der Antidiskriminierungsstelle des Bundes. Berlin: Antidiskriminierungsstelle des Bundes. Die Expertise gibt einen fundierten Überblick über die Situation behinderter Menschen am Arbeitsmarkt in Deutschland und benennt vorhandene Unterstützungsmöglichkeiten. Verwendet wurden überwiegend qualitative Methoden empirischer Sozialforschung.

Lorant et al. 107 Study Group (2017). Optimal Network for Patients with Severe Mental Illness: A Social Network Analysis. Administration and Policy in Mental Health, 44 (6), S. 877-887. Der Beitrag behandelt 
Netzwerkstrukturen in der Gesundheitsversorgung und untersucht den Einfluss unterschiedlicher Strukturmerkmale von Netzwerken (etwa Zusammensetzung, Größe, Zentralität) in Bezug auf die Ermöglichung von Versorgungskontinuitäten und sozialer Integration in Belgien. Datengrundlage ist eine Befragung von 954 in Belgien lebenden Patientinnen und Patienten.

Morgan, M., Patrick, D. L. \& Charlton, J. R. (1984). Social networks and psychosocial support among disabled people. Social Science \& Medicine, 19 (5), S. 489-97. Der Artikel befasst sich in erster Linie mit dem Einfluss von Netzwerkeigenschaften (Netzwerkgröße und Netzwerktypus) auf die Zugänglichkeit psychosozialer Unterstützung in London von Menschen mit physischer Behinderung. Er behandelt überdies Netzwerkdifferenzen nach Grad der Behinderung, wohnortanhängige Netzwerkunterschiede behinderter Menschen sowie die Bedeutung familialer Bindungen für die Verfügbarkeit emotionaler Unterstützung.

\section{Datensätze}

In Deutschland sind nur wenige Datensätze verfügbar, die Informationen über Behinderung und Erwerbsarbeit bereithalten und sich zugleich nicht nur auf wenige Basisangaben über soziale Kontakte beschränken. Erwähnenswerte Ausnahmen sind „Gesundheit in Deutschland aktuell“ (GEDA) und die „Repräsentativbefragung zur Teilhabe von Menschen mit Behinderung“.

- GEDA ist eine seit 2008 vom Robert Koch-Institut regelmäßig durchgeführte, repräsentative Querschnittsbefragung. In der letzten Erhebungswelle 2014/15 wurden 20.000 Personen in Deutschland ab 15 Jahre befragt. Enthalten sind u. a. Fragen über Behinderung, die Beschäftigungssituation, die Nutzung von Gesundheitsdiensten und über soziale Kontakte mit verschiedenen Personenkreisen und Vertrauensverhältnisse.

Weitere Informationen: https://www.rki.de/DE/Content/Gesundheitsmonitoring/Studien/Geda/Geda_node.html

- Die „Repräsentativbefragung zur Teilhabe von Menschen mit Behinderung“ wird von 2018 bis 2020 vom Institut für angewandte 
Sozialwissenschaft unter 27,000 Menschen durchgeführt. Sie beinhaltet Fragen zu Behinderung, zu Beschäftigung und Erwerbserfahrungen, zur Nutzung verschiedener wohlfahrtsstaatlicher Einrichtungen sowie zu sozialen Beziehungen und Kontakten mit Menschen mit und ohne Behinderung.

Weitere Informationen: https://www.bmas.de/DE/Service/Medien/Publikationen/Forschungsberichte/Forschungsberichte-Teilhabe/fb-492-repraesentativbefragung-behinderung.html

\section{Literatur}

Badura, B. (2008). Sozialkapital: Grundlagen von Gesundheit und Unternehmenserfolg. Berlin: Springer.

Batinic, B., Selenko, E., Stiglbauer, B., \& Paul, K. (2010). Are workers in high-status jobs healthier than others? Assessing Jahodas latent benefits of employment in two working populations. Work and Stress, 24, 73-87.

Bengtsson, S., \& Datta Gupta, N. (2017). Identifying the effects of education on the ability to cope with a disability among individuals with disabilities. PLOS ONE, 12(3), e0173659.

Benitez-Silva, H., Buchinsky, M., Chan, H. M., Rust, J., \& Cheidvasser, S. (2004). How large is the bias in self-reported disability? Journal of Applied Econometrics, 19(6), 649-670.

Borgatti, S. P., Everett, M. G., \& Johnson, J. C. (2018). Analyzing social networks. London: Sage.

Bourdieu, P. (2005). Die verborgenen Mechanismen der Macht. Hamburg: VSA.

Brucker, D. L. (2015). Social capital, employment and labor force participation among persons with disabilities. Journal of Vocational Rehabilitation, 43, 17-31.

Bundesagentur für Arbeit, Statistik/Arbeitsmarktberichterstattung. (2018). Situation schwerbehinderter Menschen. Nürnberg: Bundesagentur für Arbeit. https://statistik. arbeitsagentur.de/Navigation/Statistik/Arbeitsmarktberichte/Personengruppen/Personengruppen-Nav.html. Zugegriffen: 13. Febr. 2019.

Bundesministerium für Arbeit und Soziales [BMAS]. (2011). Übereinkommen der Vereinten Nationen über Rechte von Menschen mit Behinderungen. Erster Staatenbericht der Bundesrepublik Deutschland. Vom Bundeskabinett beschlossen am 3. August 2011. https://www.bmas.de/SharedDocs/Downloads/DE/staatenbericht-2011.pdf?__blob=publicationFile. Zugegriffen: 13. Febr. 2019.

Chronister, J., Chou, C. C., Frain, M., \& da Silva Cardoso, E. (2008). The relationship between social support and rehabilitation related outcomes: A meta-analysis. Journal of Rehabilitation, 74(2), 16-32. 
Deutsche Gesetzliche Unfallversicherung e.V. [DGUV]. (2014). Leitfaden zum Betrieblichen Eingliederungsmanagement - Praxishilfe für die Beratung der UV-Träger in den Betrieben. http://publikationen.dguv.de/dguv/pdf/10002/12263.pdf. Zugegriffen: 04. April 2018.

Diewald, M., \& Sattler, S. (2010). Soziale Unterstützungsnetzwerke. In C. Stegbauer (Hrsg.), Netzwerkforschung (S. 689-799). Wiesbaden: Springer VS.

Deutsches Studentenwerk. (2013). Studium und Behinderung - Informationen für Studierende und Studieninteressierte mit Behinderungen und chronischen Krankheiten. Berlin: Bundesministerium für Bildung und Forschung. https://www.studentenwerke.de/ sites/default/files/37_handbuch_studium_und_behinderung_7_auflage.pdf. Zugegriffen: 13. Febr. 2019.

Eichhorst, W., Kenzia, M. J., Knudsen, J. B., Hansen, M. O., Vandeweghe, B., Vanhoren, I., Rückert, E., \& Schulte, B. (2010). The Mobility and Integration of People with Disabilities into the Labour Market. IZA Research Report, 29. http://legacy.iza.org/en/webcontent/publications/reports/report_pdfs/iza_report_29.pdf. Zugegriffen: 13. Febr. 2019.

Engels, D., Engel, H. \& Schmitz, A. (2017). Teilhabebericht der Bundesregierung über die Lebenslagen von Menschen mit Beeinträchtigungen. Köln: ISG Institut für Sozialforschung und Gesellschaftspolitik. http://www.bmas.de/SharedDocs/Downloads/ DE/PDF-Publikationen/a125-16-teilhabebericht.pdf?__blob=publicationFile\&v=9. Zugegriffen: 13. Febr. 2019.

Escorpizo, R., Reneman, M. F., Ekholm, J., Fritz, J., Krupa, T., \& Marnetoft, S.-U. (2011). A conceptual definition of vocational rehabilitation based on the ICF: Building a shared global model. Journal of Occupational Rehabilitation, 21, 126-133.

Famira-Mühlberger, U., Huemer, U., \& Mayrhuber, C. (2015). Der Einfluss der sozialen Sicherungssysteme auf die Beschäftigungsquote Älterer in traditionellen Wohlfahrtsstaaten, WIFO Working Papers, No. 499, Austrian Institute of Economic Research (WIFO), Vienna.

Forrester-Jones, R., Carpenter, J., Coolen-Schrijner, P., Cambridge, P., Tate, A., Beecham, J., \& Wooff, D. (2006). The social networks of people with learning disabilities living in the community twelve years after resettlement from long-stay hospitals. Journal of Applied Research in Intellectual Disabilities, 19, 285-295.

Granovetter, M. (1973). The strength of weak ties. American Journal of Sociology, 6, 1360-1380.

Granovetter, M. (1995). Getting a job. A study of contacts and careers. Chicago: The University of Chicago Press.

Hammerschmidt, M. (1992). Behindertenverbände im sozialpolitischen Entscheidungsprozeß. Frankfurt a. M.: New York Campus.

Hinz, T. \& Abraham, M. (2008). Theorien des Arbeitsmarktes: Ein Überblick. In M. Abraham \& T. Hinz (Hrsg.), Arbeitsmarktsoziologie. Probleme, Theorien, empirische Befunde. Wiesbaden. Springer VS.

Jahoda, M. (1982). Employment and unemployment: a social-psychological analysis. Cambridge: Cambridge University Press.

Jahoda, M. (1983). Wieviel Arbeit braucht der Mensch?. Weinheim: Beltz.

Kastl, J. M. (2017). Einführung in die Soziologie der Behinderung. Wiesbaden: Springer VS. 
Klemm, K. (2015). Inklusion in Deutschland. Daten und Fakten. Gütersloh: Bertelsmann Stiftung.

Knox, M., \& Parmenter, Trevor R. (1993). Social networks and support mechanisms for people with mild intellectual disability in competitive employment. International of Rehabilitation Research, 16, 1-12.

Kohl, S., \& Niehaus, M. (2014). Inklusive Gestaltung betrieblicher Gesundheitsmaßnahmen für junge und ältere Mitarbeiterinnen und Mitarbeiter durch die Schwerbehindertenvertretung als proaktiver betrieblicher Initiator. In e.V. GfA, (Hrsg.), 60. Kongress der Gesellschaft für Arbeitswissenschaft (GfA) e.V. Gestaltung der Arbeitswelt der Zukunft (S. 647-649). Dortmund: GfA-Press.

Kohl, S., Niehaus, M., \& Baumann, A. (2015). Die UN-Behindertenrechtskonvention und die Folgen für die Arbeit mit Schwerbehindertenvertretungen (SBV) in Betrieben und Dienststellen. In A. Leonhardt (Hrsg.), Die UN-Behindertenrechtskonvention und ihre Umsetzung. Beiträge zur interkulturellen und international vergleichenden Heil- und Sonderpädagogik (S. 572-579). Bad Heilbrunn: Klinkhardt.

Lang, F. R. (2003). Die Gestaltung und Regulation sozialer Beziehungen im Lebenslauf: Eine entwicklungspsychologische Perspektive. Berliner Journal für Soziologie, 13(2), $175-195$.

Lewicki, A. (2014). Allgemeines Gleichbehandlungsgesetz: Zwischenbilanz eines brüchigen Konsenses. Aus Politik und Zeitgeschichte, 13-14, 21-27.

Lin, N. (2009). Social Capital. A Theory of Social Structure and Action. Cambridge: Cambridge University Press.

Lorant, V., Nazroo, J., Nicaise, P., Title107 Study Group. (2017). Optimal network for patients with severe mental illness: A social network analysis. Administration and Policy in Mental Health, 44(6), 877-887.

McKenna, M. A., \& Power, P. W. (2000). Engaging the African American family in the rehabilitation process: An intervention model for rehabilitation counselors. Journal of Applied Rehabilitation Counseling, 31, 12-18.

Morgan, M., Patrick, D. L., \& Charlton, J. R. (1984). Social networks and psychosocial support among disabled people. Social Science and Medicine, 19(5), 489-497.

Niehaus, M. (1993). Behinderung und sozialer Rückhalt. Zur sozialen Unterstützung behinderter Frauen. Frankfurt a. M.: Campus.

Niehaus, M. (2001). Geschlechtsspezifische Ansätze in der Rehabilitationsforschung. Zur Partizipation von Frauen mit Behinderungen. In A. Kämmerer \& A. Franke (Hrsg.), Klinische Psychologie der Frau. Ein Lehrbuch (S. 737-749). Göttingen: Hogrefe.

Niehaus, M., Marfels, B., Vater, G., Magin, J., \& Werkstetter, E. (2008). Betriebliches Eingliederungsmanagement: Studie zur Umsetzung des Betrieblichen Eingliederungsmanagements nach § 84 Abs. 2 SGB IX. Köln: Forschungsbericht 374 Sozialforschung. http://www.bmas.de/SharedDocs/Downloads/DE/PDF-Publikationen/f374-forschungsbericht.pdf?_blob=publicationFile. Zugegriffen: 13. Febr. 2019.

Niehaus, M., Kaul, T., Friedrich-Gärtner, L., Klinkhammer, D., \& Menzel, F. (2012). Zugangswege junger Menschen mit Behinderung in Ausbildung und Beruf. Bonn: Bundesministerium für Bildung und Forschung. https://www.fachportal-paedagogik.de/ literatur/vollanzeige.html?FId=988603\#vollanzeige. Zugegriffen: 13. Febr. 2019.

Niehaus, M., \& Saupe-Heide, M. (2012). Selbstbestimmung in der beruflichen Rehabilitation: Rechtlich verankert - In der Praxis vernachlässigt? Forum D - Entwicklungen und Reformvorschläge. Diskussionsbeitrag 15/2012. Heidelberg: Deut- 
sche Vereinigung für Rehabilitation. http://www.reha-recht.de/fileadmin/download/ foren/d/2012/D15-2012_Selbstbestimmung_in_beruflicher_Reha.pdf. Zugegriffen: 13. Febr. 2019.

Niehaus, M. \& Bauer, J. (2013). Chancen und Barrieren für hochqualifizierte Menschen mit Behinderung. Übergang in ein sozialversicherungspflichtiges Beschäftigungsverhältnis. Aktion Mensch. https://promi.uni-koeln.de/wp-content/uploads/2014/03/ Niehaus-Bauer-2013-Chancen-und-Barrieren-fuer-hochqualifizierte-Menschen-mit-Behinderung-Abschlussbericht.pdf. Zugegriffen: 13. Februar 2019.

Niehaus, M., \& Vater, G. (2014). Psychische Erkrankungen und betriebliche Wiedereingliederung. WSI Mitteilungen, 7, 374-377.

Pfaff, H. (2012). Lebenslagen der behinderten Menschen - Ergebnisse des Mikrozensus 2009. Wirtschaft und Statistik. Wiesbaden: Statistisches Bundesamt. https://www. destatis.de/DE/Publikationen/WirtschaftStatistik/Sozialleistungen/Lebenslagenbehinderte032012.pdf?_blob=publicationFile. Zugegriffen: 13. Februar 2019.

Potts, B. (2005). Disability and employment: Considering the importance of social capital. Journal of Rehabilitation, 71(3), 20-25.

Putnam, R. D. (2000). Bowling alone. The collapse and revival of American community. New York: Simon \& Schuster.

Rauch, A. (2005). Behinderte Menschen auf dem Arbeitsmarkt. In R. Bieker (Hrsg.), Teilhabe am Arbeitsleben. Wege der beruflichen Integration von Menschen mit Behinderung (S. 25-43). Stuttgart: Kohlhammer.

Reims, N., Nivorozhkin, A. \& Tophoven, S. (2017). Personen mit gesundheitlichen Einschränkungen - Berufliche Rehabilitation zielt auf Prävention und passgenaue Förderung. IAB-Kurzbericht, 25. http://doku.iab.de/kurzber/2017/kb2517.pdf. Zugegriffen: 04. April 2018.

Reims, N., Tisch, A., \& Tophoven, S. (2016). Junge Menschen mit Behinderung: Reha-Verfahren helfen beim Berufseinstieg. IAB-Kurzbericht, 7. http://doku.iab.de/kurzber/2016/ kb0716.pdf. Zugegriffen: 04. Apr. 2018.

Riedel, H.-P., Schmidt, C., Ellger-Rüttgard, S., Karbe, H., Niehaus, M., Rauch, A., Schian, H.-M., Schmid, S., Schott, T., Schröder, H., Spijkers, W., \& Wittwer, U. (2009). Die Zukunft der beruflichen Rehabilitation Erwachsener gestalten: Acht Handlungsfelder als Ausgangspunkt für einen akteursübergreifenden Innovationsprozess. Die Rehabilitation, 48(6), 375-382.

Rohrmann, E. (2012). Zwischen selbstbestimmter sozialer Teilhabe und fürsorglicher Ausgrenzung: Lebenslagen und Lebensbedingungen von Menschen, die wir behindert nennen. In E.-U. Huster, J. Boeckh, \& H. Mogge-Grotjahn (Hrsg.), Handbuch Armut und soziale Ausgrenzung (S. 619-642). Wiesbaden: Springer VS.

Schröder, H., Knerr, P. \& Wagner, M. (2009). Vorstudie zur Evaluation von Maßnahmen zur Förderung der Teilhabe behinderter Menschen und schwerbehinderter Menschen am Arbeitsleben. Bonn: infas.

Schröttle, M., Hornberg, C., Glammeier, S., Sellach, B., Kavemann, B., Puhe, H., \& Zinsmeister, J. (2013). Lebenssituation und Belastungen von Frauen mit Behinderungen und Beeinträchtigungen in Deutschland. Berlin: Bundesministerium für Familie, Senioren, Frauen und Jugend. 
Schröttle, M., Hornberg, C., Zapfel, S., Wattenberg, I., Vogt, K., Kellermann, G., \& Becker, J. (2014). Vorstudie für eine Repräsentativbefragung zur Teilhabe von Menschen mit Behinderung(en). Berlin: Bundesministerium für Arbeit und Soziales. http://www.bmas. de/SharedDocs/Downloads/DE/PDF-Publikationen/forschungsbericht-vorstudie-repraesentativbefragung-zur-teilhabe-von-menschen-mit-behinderung.pdf?_blob=publicationFile. Zugegriffen: 13. Febr. 2019.

von Kardorff, E. (2010). Soziale Netzwerke in der Rehabilitation und im Gesundheitswesen. In C. Stegbauer \& R. Häußling (Hrsg.), Handbuch Netzwerkforschung (S. 715724.). Wiesbaden: Springer VS.

von Kardorff, E., Ohlbrecht, H. \& Schmidt, S. (2013). Zugang zum allgemeinen Arbeitsmarkt für Menschen mit Behinderungen. Expertise im Auftrag der Antidiskriminierungsstelle des Bundes. https://www.antidiskriminierungsstelle.de/SharedDocs/ Downloads/DE/publikationen/Expertisen/Expertise_Zugang_zum_Arbeitsmarkt.pdf? blob=publicationFile. Zugegriffen: 13. Februar 2019.

UN-Behindertenrechtskonvention [UN-BRK]. (2008). Gesetz zu dem Übereinkommen der Vereinten Nationen vom 13. Dezember 2006 über die Rechte von Menschen mit Behinderungen sowie zu dem Fakultativprotokoll vom 13. Dezember 2006 zum Übereinkommen der Vereinten Nationen über die Rechte von Menschen mit Behinderungen. Bundesgesetzblatt Teil II Nr. 35. http://www.un.org/depts/german/uebereinkommen/ar61106-dbgbl.pdf. Zugegriffen: 13. Febr. 2019.

Wansing, G. \& Westphal, M. (2014). Behinderung und Migration. Kategorien und theoretische Perspektiven. In G. Wansing \& M. Westphal (Hrsg.), Behinderung und Migration. Inklusion, Diversität, Intersektionalität (S. 17-47). Wiesbaden: Springer VS.

Weber, A., \& Weber, U. (2015). Älter, hörbeeinträchtigt und... erwerbstätig! Journal of Labour Market Research, 48(3), 263-270.

Weinbach, C. (2014). Von personalen Kategorien zu Sozialstrukturen. In G. Wansing \& M. Westphal (Hrsg.), Behinderung und Migration - Inklusion, Diversität, Intersektionalität (S. 73-82). Wiesbaden: Springer VS.

Weller, S. I. (2017). Tätigkeiten Erwerbstätiger mit Behinderung - Eine empirische Anwendung des tätigkeitsbasierten Ansatzes für die Beschreibung von Arbeitsplätzen von Erwerbstätigen mit Behinderung in Deutschland. Berichte zur beruflichen Bildung. Bonn: Bundesinstitut für Berufsbildung.

Welti, F. (2005). Behinderung und Rehabilitation im sozialen Rechtsstaat. Freiheit, Gleichheit und Teilhabe behinderter Menschen. Tübingen: Mohr Siebeck.

Wuppinger, J. \& Rauch, A. (2010). Wiedereingliederung in den Arbeitsmarkt im Rahmen beruflicher Rehabilitation: Maßnahmeteilnahme, Beschäftigungschancen und Arbeitslosigkeitsrisiko. IAB-Forschungsbericht. http://doku.iab.de/forschungsbericht/2010/ fb0110.pdf. Zugegriffen: 13. Febr. 2019.

Zapfel, S. (2015). Berufliche Rehabilitation und Return to Work von Personen mit Migrationshintergrund. In A. Weber, L. Peschkes, \& W. de Boer (Hrsg.), Return to WorkArbeit für alle: Grundlagen der beruflichen Reintegration (S. 239-246). Stuttgart: Genter. 
Open Access Dieses Kapitel wird unter der Creative Commons Namensnennung 4.0 International Lizenz (http://creativecommons.org/licenses/by/4.0/deed.de) veröffentlicht, welche die Nutzung, Vervielfältigung, Bearbeitung, Verbreitung und Wiedergabe in jeglichem Medium und Format erlaubt, sofern Sie den/die ursprünglichen Autor(en) und die Quelle ordnungsgemäß nennen, einen Link zur Creative Commons Lizenz beifügen und angeben, ob Änderungen vorgenommen wurden.

Die in diesem Kapitel enthaltenen Bilder und sonstiges Drittmaterial unterliegen ebenfalls der genannten Creative Commons Lizenz, sofern sich aus der Abbildungslegende nichts anderes ergibt. Sofern das betreffende Material nicht unter der genannten Creative Commons Lizenz steht und die betreffende Handlung nicht nach gesetzlichen Vorschriften erlaubt ist, ist für die oben aufgeführten Weiterverwendungen des Materials die Einwilligung des jeweiligen Rechteinhabers einzuholen.

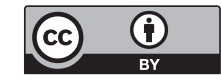

